\title{
Development and preliminary validation of the work-unit performance questionnaire
}

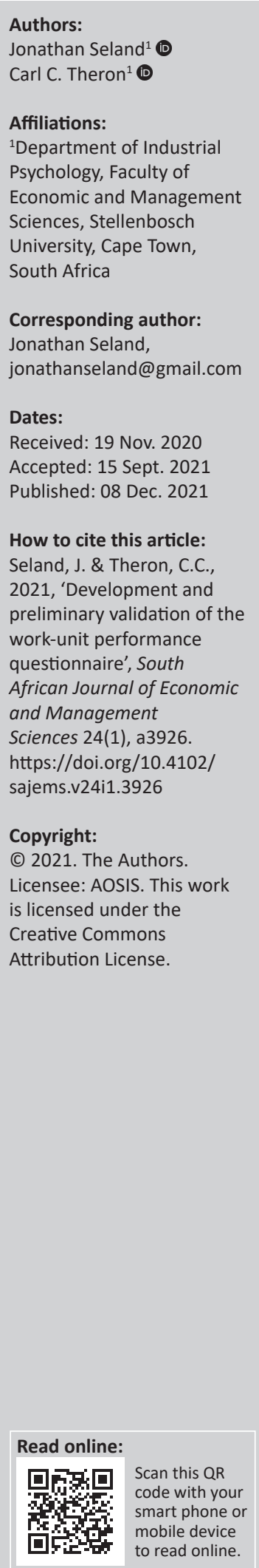

Authors:

Affiliations

${ }^{1}$ Department of Industria Psychology, Faculty of

Sciences, Stellenbosch

University, Cape Town

South Africa

Jonathan Seland,

Accepted: 15 Sept. 2021

ublished: 08 Dec. 202

this article:

Seland, J. \& Theron, C.C.

preliminary validation of the

African Journal of Economic

and Management

https://doi.org/10.4102/

sajems.v24i1.3926

Copyright:

Licensee: AOSIS. This work

is licensed under the

Creative Commons

mobile device
to read online.
Background: Serious shortcomings are identified in the Performance Index (PI), developed by Spangenberg and Theron (2004). Attempts are made to correct these shortcomings.

Aim: The primary objective of this research study was to develop and preliminary validate a generic Work-Unit Performance Questionnaire, based on the Performance Index of Spangenberg and Theron (2004), correcting shortcomings identified in that particular PI.

Setting: The study used convenience sampling that consisted of 202 respondents from a variety of South African industries. The article draws on findings in the thesis of Seland (2019).

Methods: The development and preliminary validation of the Work-Unit Performance Questionnaire (WUPQ) was required. The WUPQ consists of dual subscales, the Work-Unit Competency Questionnaire (WUCQ), which consists of seven latent behavioural competency variables, and the Work-Unit Outcome Questionnaire (WUOQ), which consists of six latent outcome variables.

Results: Both measurement models (WUCP \& WUOQ) showed close fit; however, two factor loadings in the WUCQ measurement model had to be constrained. Reasonable structural model fit was found in the sample. Support was found for 11 of the original 21 path-specific substantive hypotheses and for an additional hypothesis.

Conclusion: The proposed model can be used by managers, with caution due to the intentional exclusion of competency potential and situational variables, to diagnose poor work-unit performance. Furthermore, it is encouraged that this research be the starting point for further analyses of work-unit performance and advance validation of the instrument.

Keywords: Work-unit performance; Work-Unit Performance Questionnaire; industrial psychology; structural equation modelling; measurement model; SPSS; LISREL; Psychometrics.

\section{Introduction}

Organisations consist of many collaborative activities that can only be performed through a system of connected departments in order to function efficiently and effectively, and to obtain the triple bottom line of generating profit, serving people and the planet (Elkington 1998; LopezCabrales \& Valle-Cabrera 2020; Slaper \& Hall 2011). To obtain the central goals of an organisation, these aforementioned departments must operate in an efficient and effective manner. One of these functions is the human resource department (HR).

The impact of HR through various interventions and labour practices, is vital to the attainment of the triple bottom line. This impact of HR interventions and labour practices is based on the knowledge that human capital is the most important asset in an organisation and all other factors of production rely on the performance of the workforce (Kaur \& Kaur 2017; Theron 1999).

Historically, regular HR interventions seem to have largely, although not exclusively, focussed on enhancing the performance of individual employees and, to some degree, also on the enhancement of team performance.

Regular HR interventions aimed at enhancing the performance of work units, however, do not seem to be the norm. In the current study it would be argued that dedicated HR interventions are also required to monitor and enhance work-unit performance. Although there has been a great amount of research on team work, a complete and agreed-upon set of factors in terms of which team performance can be conceptualised, has not been identified (Andersson, Rankin \& Diptee 
2017). A work unit can, however, not be equated with a team. The current study used a generic definition, and defined a work unit as a temporary or permanent organisational entity that operates in a private, state-owned or not-for-profit organisation. The size of these work units varies from a small team, consisting of a leader and three subordinates, to a department within a company that is comprised of a large number of individuals (Spangenberg \& Theron 2004).

Work-unit performance, similar to the performance of individual employees, is generically defined as the nomological network of structural relations existing between an interrelated set of latent behavioural performance dimensions and an interrelated set of latent outcome variables, valued by the organisation, which contribute to organisational goals (Myburgh \& Theron 2014). However, these should now be interpreted as the synergetic combination of the behaviour and achievements of its members and not of the individual employee. The organisational work unit is dependent on the action of its members and, although work units consist of individuals, the combination of the performance by its individual members does not necessarily equate to the level of performance by the work unit. It is acknowledged that adequate work-unit performance requires a satisfactory performance of each of its members. However, a synergy exists within a work unit, and individually talented members do not guarantee performance from the entire work unit. The effectiveness and efficiency of the unit is greater than the sum of its parts. There are many pertinent cases of units, composed of members with average capacity, that achieve outstanding results.

A multifaceted nomological network of latent variables that constitute the unit and the wider organisational context, is responsible for the level of functioning in the work unit. In order to improve the performance of the work unit, it is essential to identify what constitutes work-unit performance; the causes of work-unit performance are recognised, the way in which they structurally combine is conceptualised, and a psychometrically sound instrument to measure work-unit performance has been developed. We regard the limited knowledge of the performance of the work unit and its determinants as a major shortcoming in industrial psychology literature. Organisations are essentially an agglomeration of work units that work towards a shared goal (Spangenberg \& Theron 2004). Each work unit in an organisation needs to operate efficiently and effectively to achieve this goal. It is unlikely that an individual, or collection of individuals working alone, could run all of the operations that an organisation needs to function optimally.

Using the research done by Nicholson and Brenner (1994) and Cockerill, Schroder and Hunt (1993) as a foundation, Spangenberg and Theron (2004) have been at the forefront of investigations into the conceptualisation and operationalisation of universal work-unit performance. Therefore, they form the foundation of this research. The Nicholson and Brenner (1994) theory of work-unit performance consisted of four work-unit performance dimensions: namely, wealth of resources, markets, adaptability and climate. Cockerill et al. (1993) further conceptualised four work-unit performance dimensions: output, climate, adaptability and resource input. Spangenberg and Theron (2004) suggested that the aforementioned studies did not explain the full connotative meaning of the unit performance construct and proposed eight performance dimensions. Furthermore, they developed a generic measure of organisational-unit performance, the Performance Index (PI) (Henning, Spangenberg \& Theron 2004).

Henning et al. (2004) further contributed to the conceptualisation of the work-unit performance construct by developing and empirically testing a structural model that explains the way in which the work-unit performance dimensions, directly and indirectly, impact one another. Unfortunately, very little research had been done on the PI and its conceptualisation of the work-unit performance construct since Henning et al. (2004) developed and empirically tested the original structural model. Dunbar, Theron and Spangenberg (2011) successfully cross-validated the PI via a multigroup confirmatory factor analytic study. Theron and Spangenberg (2016) empirically tested the structural invariance of the Henning et al. (2004) structural model. Theron and Spangeneberg (2016) found gammacs, beta ${ }^{\mathrm{CS}}$, $\mathrm{psi}^{\mathrm{CS}}$, and $\mathrm{phi}^{\mathrm{CS}}$ invariance and gamma ${ }^{\mathrm{CS}}$, beta ${ }^{\mathrm{CS}}$, $\mathrm{psi}^{\mathrm{CS}}$, and $\mathrm{phi}^{\mathrm{CS}}$ equivalence. The structural model of Henning et al. (2004), therefore, cross-validated successfully. Swart (2013) used the PI in her study on the development and empirical evaluation of a comprehensive leadership-unit performance structural model.

However, the authors of this article propose that the Spangenberg and Theron (2004) conceptualisation fails to provide an adequate explanation of the organisational workunit performance construct. The Spangenberg and Theron (2004) notion of the organisational work-unit construct appears to inappropriately include a number of latent variables from the latent organisational-unit competency potential variable domain. Competency potential latent variables refer to the individual characteristics required for an individual to display the prescribed behaviours (Bartram 2006; Slabbert \& Hoole 2021).

They furthermore appear to correctly incorporate latent behavioural competencies and latent outcome variables. However, they do not completely represent these domains. In accordance to SHL (2000) and Bartram $(2005,2006)$, latent behavioural competencies are the abstract theme in related sets of behaviours that are instrumental in the delivery of the results or outcomes for which the unit exists. Latent outcome variables, according to Bartram (2006), as well as Slabbert and Hoole (2021), refer to the actual, or intended outcomes of behaviour, which have been defined, either explicitly or implicity, by the individuals, their line manager, or the organisation. The Henning et al. (2004) PI model does not fully differentiate the competency and outcome domains. This shortfall exists, because Spangenberg and Theron (2004) did not consider the phenomenon from a competency 
modelling perspective. The term competency modelling is used in this context to refer to the development and empirical testing of a structural model that describes specific structural relationships amongst competency potential latent variables, situational latent variables, competency latent variables and outcome latent variables. This interpretation goes significantly beyond the conventional interpretations (SHL 2011; Slabbert \& Hoole 2021). The question could justifiably be raised why these shortcomings were not appreciated and corrected earlier by Theron or Spangenberg? The question could also be asked why nobody else raised concerns, regarding the conceptualisation of the work-unit performance construct, proposed by Spangenberg and Theron (2004). Disappointing as both of these inattentions are, they cannot be used to argue, or imply, that the aforementioned shortcomings are not serious and do warrant attempts to correct them.

Quite often the truth is staring one in the face, without one appreciating the fact. But when the proverbial penny finally drops, the shortcomings need to be acknowledged frankly, and sincere steps taken to correct them. The current study constitutes a practical attempt to do just that. It attempts to correct the PI's conceptualisation of work-unit performance by removing the work-unit competency potential variables from the model, and adding the omitted latent competencies and outcomes to the current model. This necessitates the creation and psychometric evaluation of a revised PI [the Work-Unit Performance Questionnaire (WUPQ)]. This revised version consists of two subscales, the Work-Unit Competency Questionnaire (WUCQ) and the Work-Unit Outcome Questionnaire (WUOQ).

\section{Research objectives}

Based on this argument, the current study had the overarching objective to (re-)conceptualise and (re-)operationalise the organisational work-unit performance construct. The overarching objective was dissected into the operational objectives listed below:

- Clarify the connotative meaning of the work-unit performance construct (this necessitates the development of a structural model explicating the structural relationships between latent behavioural unit competencies and latent unit outcomes).

- Explore the denotative meaning of the work-unit performance construct by operationally defining the latent behavioural unit competencies and latent unit outcomes.

- Create an instrument to measure work-unit competencies (WUCQ).

- Preliminarily assess the psychometric properties of the WUCQ by fitting the relevant measurement model.

- Create an instrument to measure work-unit performance outcome (WUOQ).

- Preliminarily assess the psychometric properties of the WUOQ by fitting the relevant measurement model.

- Fit a structural model, which depicts the structural relations existing between the latent behavioural workunit competencies and latent work-unit outcomes, according to the constitutive definition of the work-unit performance construct, to preliminarily evaluate the construct validity of the WUCQ and WUOQ.

\section{Clarifying the work unit performance construct}

Concise definitions of the performance dimensions conceptualised to constitute the performance construct in the PI are shown in Table 1.

The cumulative research by Spangenberg and Theron (2004) on the PI formed the foundation for the re-conceptualisation of the organisational work-unit performance construct. In an attempt to rectify the limitations of the PI and further knowledge on work-unit performance, the dimensions of the PI were categorised into one of the three domains in a competency model. Thereafter, an attempt was made to identify the limitations in the behavioural competencies and outcome domains.

Spangenberg and Theron (2004), evidently in Table 1, did not fully consider competency potential variables in their conceptualisation. It is acknowledged that their work-unit competency model was not created with the purpose to be comprehensive in nature, but rather to illustrate the inner workings of the work-unit performance construct.

TABLE 1: The definitions of the Performance Index dimensions and the categorisation of the Performance Index dimensions into domains.

\begin{tabular}{|c|c|c|}
\hline Dimension & Domain & Definition \\
\hline $\begin{array}{l}\text { Production and } \\
\text { efficiency }\end{array}$ & $\begin{array}{l}\text { Outcome latent } \\
\text { variable }\end{array}$ & $\begin{array}{l}\text { Quantitative outputs such as meeting } \\
\text { goals, quantity, quality and cost- } \\
\text { effectiveness, and task performance. }\end{array}$ \\
\hline $\begin{array}{l}\text { Core people } \\
\text { processes }\end{array}$ & $\begin{array}{l}\text { Behavioural } \\
\text { competency }\end{array}$ & $\begin{array}{l}\text { Organisational effectiveness criteria such } \\
\text { as goals and work plans, communication, } \\
\text { organisational interaction, conflict } \\
\text { management, productive clashing of } \\
\text { ideas, integrity and uniqueness of the } \\
\text { individual or group, learning through } \\
\text { feedback and rewarding performance. }\end{array}$ \\
\hline Work-unit climate & $\begin{array}{l}\text { Outcome latent } \\
\text { variable }\end{array}$ & $\begin{array}{l}\text { Psychological environment of the unit, } \\
\text { and gives an overall assessment of the } \\
\text { integration, commitment and cohesion of } \\
\text { the unit. It includes working atmosphere, } \\
\text { teamwork, work group cohesion, } \\
\text { agreement on core values and consensus } \\
\text { regarding the vision, achievement-related } \\
\text { attitudes and behaviours and } \\
\text { commitment to the unit. }\end{array}$ \\
\hline $\begin{array}{l}\text { Employee } \\
\text { satisfaction }\end{array}$ & $\begin{array}{l}\text { Outcome latent } \\
\text { variable }\end{array}$ & $\begin{array}{l}\text { Satisfaction with the task and work } \\
\text { context, empowerment, and career } \\
\text { progress, as well as with outcomes of } \\
\text { leadership. }\end{array}$ \\
\hline Adaptability & $\begin{array}{l}\text { Competency potential } \\
\text { latent variable }\end{array}$ & $\begin{array}{l}\text { Flexibility of the unit's management and } \\
\text { administrative systems, core processes } \\
\text { and structures, capability to develop new } \\
\text { products or services and versatility of staff } \\
\text { and technology. Overall, it reflects the } \\
\text { capacity of the unit to appropriately and } \\
\text { expeditiously change. }\end{array}$ \\
\hline Capacity & $\begin{array}{l}\text { Competency potential } \\
\text { latent variable }\end{array}$ & $\begin{array}{l}\text { Internal strength of the unit, financial } \\
\text { resources, profits and investment, } \\
\text { physical assets and materials; supply, } \\
\text { quality and diversity of staff. }\end{array}$ \\
\hline $\begin{array}{l}\text { Market share/ } \\
\text { standing }\end{array}$ & $\begin{array}{l}\text { Outcome latent } \\
\text { variable }\end{array}$ & $\begin{array}{l}\text { Market share, competitiveness and } \\
\text { market-directed diversity of products or } \\
\text { services, customer satisfaction and } \\
\text { reputation for adding value to the } \\
\text { organisation. }\end{array}$ \\
\hline Future growth & $\begin{array}{l}\text { Outcome latent } \\
\text { variable }\end{array}$ & $\begin{array}{l}\text { Index of projected future performance, } \\
\text { including profits and market share, capital } \\
\text { investment, staff levels and expansion of } \\
\text { the unit. }\end{array}$ \\
\hline
\end{tabular}

Source: Spangenberg, H.H. \& Theron, C., 2004, 'Development of a questionnaire for assessing work unit performance', SA Journal of Industrial Psychology 30(1), 19-28. https://doi. org/10.4102/sajip.v30i1.134. 
While Spangenberg and Theron (2004) only included two competency potential variables, they were incorrectly incorporated. Therefore, only certain competency potential latent variables and limited relevant competency and outcome latent variables were included in the current PI model (Henning et al. 2004) therefore it cannot be considered comprehensive. Similarly, the current model is unsuccessful as an organisational work-unit performance model, because it erroneously includes competency potential latent variables and omits applicable work-unit competencies and outcomes. There are a vast variety of latent competency variables that are pivotal to a work unit in the attainment of relevant outcomes within the organisational context. The PI model did not consider this vast variety, and only a limited number of dimensions are classified into this domain. This domain had the most latent variables omitted. Therefore, the proposed organisational work-unit performance model aimed to rectify this deficiency through proposing additional competencies in this domain. The paths from the current PI model (Henning et al. 2004) are illustrated in Figure 1, along with the new proposed paths and competencies.

Organisational unit competencies are essential for a work unit to achieve the required outcomes. The Henning et al. (2004) model only presented certain variables in this domain and is, therefore, considered incomplete. While it had more variables than the competency domain, further research was necessary to fully characterise this domain. A competency model in organisational work-unit performance must fully acknowledge the psychological mechanisms at play and, therefore, not fully representing the variables in the competency and outcome domains is a serious shortfall in the research done by Spangenberg and Theron (2004), as well as Henning (2002). A competency model is, in part, made up of the internal structure of the performance construct. The oversight of certain variables in the organisational work-unit performance model thus reduces the capacity of a competency model to completely characterise the psychological mechanisms which are responsible for the different level of organisational work-unit performance.

\section{Additional organisational work-unit competencies and outcomes}

Organisational work units are cohesive assortments of personnel working in the attainment of a shared objective. Therefore, the performance achieved, is greater than the simple addition of the performance by the individual members. Based on this logic, the work unit operates, similarly to an employee, as a cohesive being. The position taken in this article is that it is beneficial to attribute particular competencies of the employee to the organisational work unit. Numerous performance models that illustrate individual employee performance, based on behavioural competencies, have been put forward (Bartram, Robertson \& Callinan, 2002; Campbell 1990; Campbell \& Wiernik 2015; Myburgh \& Theron 2014; Viswesweran \& Ones 2000). Specific competencies were identified from these

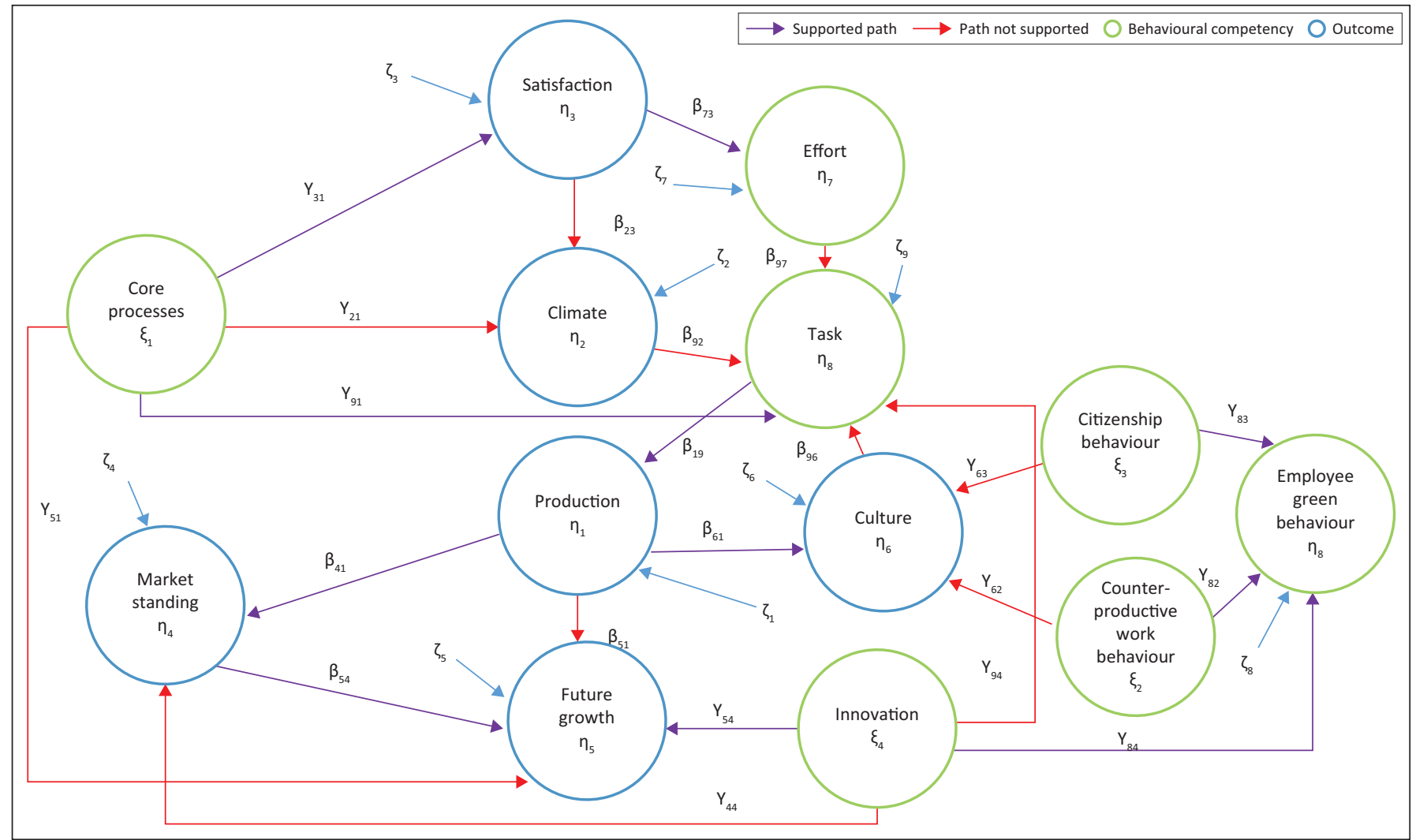

Source: Seland, J.M., 2019, 'The development and empirical testing of a revised Performance Index', Master's thesis, p. 270, Dept. of Industrial Psychology, Stellenbosch University.

Note: Satisfaction refers to satisfaction, effort refers to effort, core processes refers to core people processes, climate refers to the work-unit climate, task performance refers to task performance, citizenship behaviour refers to organisational citizenship behaviour, production refers to production and efficiency, culture refers to high performance culture, EGB refers to EGB, counterproductive work behaviour refers to counterproductive work behaviour, market standing refers to market standing, future growth refers to future growth and innovation refers to innovation.

FIGURE 1: Proposed work unit performance structural model. 
conceptualisations to be included in the organisational workunit performance construct. The motivation for the inclusion of each of the additional work-unit competencies and outcomes is provided below. Table 2 provides a concise description of the suggested latent behavioural competencies and outcomes that form part of the organisational work unit construct.

\section{Innovation}

In the 21st century the market is consistently evolving and increasingly in competition. Organisational work units must continue to be relevant and succeed in these conditions through creativity that is the foundation for innovation. More so, the great diversity in which innovation occurs in an organisational unit is especially relevant. Innovation is characterised by the conception of novel ideas, dealing with any challenges that occur, and is notably strategically orientated. These actions are especially advantageous to an organisational work unit. Thus, innovation is the first proposed competency to be included in the partial work-unit competency model.

\section{Effort}

In order to succeed, the organisation work unit must display the required effort. This effort must be enough to complete the necessary objectives, and no free-riding can take place. The definition, as described in Table 2, is significant to the present study as the concept of effort is generally associated with the giving of one's time, while the other facets are omitted. However, the construct is multi-faceted and includes aspects of time, resources and care. It is also important to note the last component in the definition, the willingness to work under detrimental conditions, is commonly referred to as grit (Duckworth et al. 2007). Grit is key to the effectiveness and efficiency of a work unit.

\section{Task performance}

The current conceptualisation of work-unit performance is flawed as it does not consider the fundamental duty of an organisational work unit, that is to transform limited resources into goods and services which the market needs. It is hoped that including the notion of task performance into the current conceptualisation of work-unit performance, will remedy this shortcoming. Operationally task performance is the combined perception, shared by the members, of the manner in which the work unit performs the activities that advance the technical core of the organisation, carries out the foundational, substantive and fundamental technical duties of the work unit, efficiently and effectively finishes tasks prescribed in the job description of the work unit and accomplishes work-unit goals.

\section{Counterproductive work behaviour}

The aforementioned competencies are positive in nature and their presence is essential to the success of a work unit. However, if members of the work unit display the behaviours in the category of counterproductive workplace behaviour (CWB), it will detrimentally impact the extent to which the unit achieves its outcomes. The constructive interaction of
TABLE 2: A summary of the seven latent behavioural competencies and six latent outcome variables included in the organisational work-unit performance construct.

\begin{tabular}{ll}
\hline Dimensions & Categorisation \\
\hline Innovation & $\begin{array}{l}\text { Behavioural } \\
\text { competency }\end{array}$ \\
\hline Effort & $\begin{array}{l}\text { Behavioural } \\
\text { competency }\end{array}$
\end{tabular}
Definition

The extent to which unit employees display creativity, not only in their individual jobs but also on behalf of the whole organisational unit, show openness to new ideas and experiences, handle novel situations and problems with innovation and creativity, think broadly and strategically in order to support and drive desired organisational unit change. The extent to which the unit employees
devote constant attention towards their work use resources like time and care spent in order to be effective on the job, show willingness to keep working under detrimental conditions and spend the extra effort required for the task.

Counterproductive Behavioural The extent to which the unit employee displays behaviour that threaten the wellbeing of an organisation, shows unwillingness to comply with organisational unit rules, interprets organisational unit
expectations incorrectly, fails to maintain expectations incorrectly, fails to maintain personal discipline, absent from work, not punctual, steals, misuses drugs, displays
confrontational attitudes towards co-workers, supervisors, and the work itself, their supervisors, and the work itself, their
behaviour hinder the accomplishment of organisational unit goals.

Organisational Behavioural $\begin{array}{ll}\text { Organisational } & \text { Behavioural } \\ \text { citizenship } & \text { competency }\end{array}$

The extent to which the unit employees display voluntary behaviour, contributing display voluntary behaviour, contributing organisational unit, volunteer to carry out task activities that are not formally part of their job description, follow organisational unit rules and procedures, endorse, support, and defend organisational unit objectives, show willingness to go the extra mile, voluntary help colleagues with work, show willingness to tolerate inconveniences and impositions of work without complaining, are actively and constructively involved in organisational unit affairs.

$\begin{array}{ll}\begin{array}{l}\text { Employee green } \\ \text { behaviour }\end{array} & \begin{array}{l}\text { Behavioural } \\ \text { competency }\end{array} \\ \begin{array}{l}\text { Production and } \\ \text { efficiency }\end{array} & \begin{array}{l}\text { Outcome latent } \\ \text { variable }\end{array} \\ \begin{array}{l}\text { Core people } \\ \text { processes }\end{array} & \begin{array}{l}\text { Behavioural } \\ \text { competency }\end{array}\end{array}$

Scalable actions and behaviours unit employees engage in, linked with and contribute to or detract from environmental sustainability.

Quantitative outputs such as meeting goals, quantity, quality and cost-effectiveness.

Work-unit effectiveness criteria such as goals and work plans are used, effective communication, frequent work unit interaction, constructive conflict management, productive clashing of ideas, integrity and uniqueness of the individual o group is valued, learning through feedback and rewarding performance.

Work-unit climate Outcome latent Psychological environment of the unit, and variable gives an overall assessment of the integration, commitment and cohesion of the unit. It includes working atmosphere, teamwork, work group cohesion, agreement on core values and consensus regarding the vision, achievement-related attitudes and behaviours and commitment to the unit.

\begin{tabular}{|c|c|c|}
\hline $\begin{array}{l}\text { Employee } \\
\text { satisfaction }\end{array}$ & $\begin{array}{l}\text { Outcome latent } \\
\text { variable }\end{array}$ & $\begin{array}{l}\text { Satisfaction with the task and work context, } \\
\text { empowerment, and career progress, as well } \\
\text { as with outcomes of leadership. }\end{array}$ \\
\hline $\begin{array}{l}\text { Market share/ } \\
\text { standing }\end{array}$ & $\begin{array}{l}\text { Outcome latent } \\
\text { variable }\end{array}$ & $\begin{array}{l}\text { Market share, competitiveness and market- } \\
\text { directed diversity of products or services, } \\
\text { customer satisfaction and reputation for } \\
\text { adding value to the organisation. }\end{array}$ \\
\hline $\begin{array}{l}\text { High performance } \\
\text { culture }\end{array}$ & $\begin{array}{l}\text { Outcome latent } \\
\text { variable }\end{array}$ & $\begin{array}{l}\text { The shared perception amongst members of a } \\
\text { unit that high and exceptional performance in } \\
\text { everything that the unit does, is the norm or } \\
\text { expectation in the organisational unit. }\end{array}$ \\
\hline Future growth & $\begin{array}{l}\text { Outcome latent } \\
\text { variable }\end{array}$ & $\begin{array}{l}\text { Index of projected future performance and } \\
\text { includes profits and market share, capital } \\
\text { investment, staff levels and expansion of the } \\
\text { unit. }\end{array}$ \\
\hline Task performance & $\begin{array}{l}\text { Behavioural } \\
\text { competency }\end{array}$ & $\begin{array}{l}\text { The extent to which the work unit effectively } \\
\text { performs activities that contribute to the } \\
\text { organisation's technical core, performs the } \\
\text { foundational, substantive and core technical } \\
\text { tasks of the work unit effectively, successfully } \\
\text { completes role activities prescribed in the } \\
\text { work unit's 'job description' and achieves } \\
\text { work-unit work objectives. }\end{array}$ \\
\hline
\end{tabular}

Source: Seland, J.M., 2019, 'The development and empirical testing of a revised Performance Index', Master's thesis, pp. 52-53, Dept. of Industrial Psychology, Stellenbosch University. 
members within a unit is pivotal to its success; therefore, interpersonal aspects of $C W B$, including confrontation, absenteeism and incorrect expectations, are highlighted.

\section{Organisational citizenship behaviour}

Organisational citizenship behaviour (OCB) is a constructive construct that must be developed in an organisational work unit. The unit must be informally considered as a 'team player' within the organisation. The majority of the members within a work unit must be prepared to go the extra mile in order to safeguard the success of their work unit and the organisation as a whole. More so, it is envisioned that the members who exhibit $O C B$ could inspire other members to succeed and act similarly.

\section{Employee green behaviour}

The acceptance of environmental, social and governance (ESG) criteria is rapidly increasing and Investors often use ESG for investment decisions (Umar, Kenourgios \& Papathanasiou 2020). Therefore, organisations' accountability to theenvironment has generated increased interest in recent times (Ones \& Dilchert 2012; Ones et al. 2015). This accountability is popular with corporate leaders, scientists and society as a whole. All of the aforementioned competency latent variables can be classified in the profit and people components of the triple bottom line. Thus, to service all aspects of the triple bottom line, employee green behaviour $(E G B)$ is proposed as an additional competency to acknowledge the duty towards the planet. EGB consists of five second-order behaviours: namely conserving, working sustainably, avoiding harm, influencing others and taking initiative.

\section{High performance culture}

High performance culture (HPC) is proposed as a further outcome latent variable in the work-unit performance model. Once all members of an organisational work unit share a similar perception of the practices and standards for the way that work is expected to be completed, it will greatly assist the work unit. A powerful common culture within a work unit will further the effectiveness and efficiency of the work unit. Similarly, a HPC fosters and grows from continual high performance of the work unit.

\section{Research methodology}

A discussion on the hypotheses is presented in (Seland 2019).

\section{Research design}

Three ex post facto correlational designs, with two indicator variables per latent variable (Seland 2019), were used in this study, as direct control over the independent variables was not possible. This inability was because the nature of the latent variables does not allow manipulation.

\section{Research participants}

The non-probability sampling technique of convenience sampling was used in the study. This was achieved through the use of Facebook, a social media website, and an individual rater assessed their work unit. The purpose of the WUPQ was to be as generic as possible and Facebook provided the opportunity to evaluate a greater diversity of work units in a variety of organisations and industries. However, the resultant sample is not an accurate representation of the intended population. The eventual sample of $N=202$ fell well below the required sample size that was needed to fit the WUCQ, WUOQ and WUPQ measurement models with individual items as originally intended.

The greatest represented sector in the sample were work units in the education sector (18.3\%). The 'other' option included non-specified industries (mining, etc.) and was the second most popular industry $(17.8 \%)$. Furthermore, the third and fourth greatest represented industries were the financial services (15.8\%), and not for profit (13.4\%), respectively. Statistics South Africa (2016) reported similar findings in the formal non-agricultural sector report. Geographically, Gauteng (74.8\%) was the province with the greatest representation and Western Cape (14.4\%) was second. Just over half $(52.5 \%)$ of the respondents reported on units that consisted of less than 10 members. Interestingly, there was a near equal proportion of raters that were subordinates $(52.5 \%)$ and managers $(47.5 \%)$.

\section{Measuring instruments/operationalisation}

The study used the PI (Spangenberg \& Theron 2004) and the Generic Performance Questionnaire (Myburgh \& Theron 2014) as templates in the layout and structure of the WUCQ and the WUOQ. The WUPQ used a 5-point scale and anchored three of the scale points (1, 3 and 5) with behavioural denotations. The full questionnaire is available in Seland (2019). Provision was made for an 'unable to respond' option. The WUPQ comprised 97 items, spread across 13 subscales.

\section{Missing values}

A total of 680 missing values were found, this constituted only $3.47 \%$ of the complete data set (19 594 data points). The future growth subscale, similar to that of Spangenberg and Theron (2004), had the most missing values. More specifically, market share (item 94) and capital investment (item 95) had the greatest number of missing values, at 62 (30.69\%) and 61 $(30.19 \%)$ respectively.

Multiple imputation (MI) (Du Toit, Du Toit \& Hawkins 2001) was chosen to treat the missing values, rather than reducing the already small sample. The study complied with the MI prerequisites as less than $30 \%$ of the datacomprised missing values; the individual responses to the items were measured on five-point Likert scales and could, therefore, permissibly be treated as continuous variables, and even though the assumption of multivariate normality was not satisfied for the WUCQ and WUOQ, the observed item variables were not excessively skewed (Muthén \& Kaplan 1985). 


\section{Data analysis}

In order to assess the WUCQ, WUOQ and WUPQ, and to evaluate the work-unit performance structural model (depicted in Figure 1), several analyses were necessary. These included item analysis, exploratory factor analysis (EFA), confirmatory factor analysis (CFA) and structural equation modelling.

\section{Item analysis}

Item analyses were conducted sequentially on each subscale. The item analysis reported in this article chose not to treat the reliability analysis as part of the item analysis. The reliability coefficient typically calculated via the SPSS Reliability procedure, and reported as part of the item analysis results, is Cronbach's alpha (Cronbach 1951). Cronbach's alpha, however, makes the assumption that the subscale of items is unidimensional and that the regression of the items on the single underlying latent variable is essentially tau equivalent (Dunn, Baguley \& Brunsden 2014). The unidimensionality assumption necessitates that the calculation of reliability coefficients should be preceded by a dimensionality analysis (Huysamen 2006). The item analysis was conducted, using the SPSS 25 Reliability Procedure (SPSS 2018). The subscales in the WUCQ and WUCQ all returned satisfactory item statistics.

\section{Dimensionality analysis}

Unrestricted principal axis factor analysis, with oblique rotation, was chosen in order to conduct the EFA on the different subscales. In situations in which factor fission was discovered in the initial EFA, a CFA was run. Eight of the 13 scales passed the unidimensionality assumption. Firstly, the assumption was not met for the effort subscale. Thus, the two effort factors were understood as giving/investing/applying the unit and as a continuous focus factor (Seland 2019). Secondly, the unidimensionality assumption was not met for the $C W B$ subscale. These were understood as non-criminal offences (CWBs that are against corporate rules) and criminal offences (CWBs that are against the law).

In the WUOQ, the unidimensionality assumption was not supported for three subscales. Firstly, employee satisfaction had to be seen as two factors, satisfaction with the quality of supervision and the satisfaction with work and surrounding. Furthermore, the two factors in HPC were identified as internal focus/emphasis and an external focus/emphasis on high performance. Lastly, an internal (product offering) focus/ evaluation and an external focus/evaluation of market standing were identified as the two market standing factors (Seland 2019). The factor fission was found to make sense theoretically on all of the subscales.

Second-order CFAs were subsequently conducted on the subscales where factor fission occurred. The loading pattern in the pattern matrix was observed in order to identify the loading of the items on the two first-order factors in the second-order measurement model. The results were statistically significant $(p<0.05)$ for all the indirect effects of the second-order factor on the relevant subscale items. This justified using each item individually in the subscales where factor fission occurred as indicators of the second-order latent competencies and outcomes measured by these subscales.

\section{Reliability analysis}

Jeffreys's Amazing Statistics Program (JASP) 0.11.1 (GossSampson 2019; Love et al. 2019) was used to calculate McDonald's $\varpi$ (McDonald 1999) in addition to Cronbach's $\alpha$ for the WUPQ subscales for which the unidimensionality assumption had been corroborated. McDonald's ๘ was favoured over Cronbach's $\alpha$ because the former makes less stringent assumptions about the nature of the regression of the subscale items on the underlying latent variable. The results are shown in Table 3.

All the subscales for which the unidimensionality assumption had been corroborated, returned satisfactory reliability coefficients. The Cronbach $\alpha$ consistently returned only marginally lower reliability estimates than McDonald's ๗.

The multidimensional McDonald's $\varpi$ and the stratified $\alpha$ (Widhiarso \& Ravand 2014) was calculated for the subscales where factor fission occurred. $\varpi$ was calculated based on the factor loadings and the measurement error variances of the items in the completely standardised solution of the fitted 2 -factor measurement model. The results are shown in Table 4.

All the WUOQ subscales for which the unidimensionality assumption had not been corroborated, returned satisfactory $\omega$ and $\alpha$ estimates. The two WUCQ subscales where factor fission was found, returned marginally satisfactory $\omega$ 's but

TABLE 3: McDonald's $\varpi$ and Cronbach's $\alpha$ for the Work-Unit Performance Questionnaire subscales for which the unidimensionality assumption had been corroborated.

\begin{tabular}{lcc}
\hline Subscale & McDonald's $\varpi$ & Cronbach's $\boldsymbol{\alpha}$ \\
\hline Subscale WUCQ & & \\
Innovation & 0.879 & 0.878 \\
Organisational Citizenship & 0.845 & 0.843 \\
Behaviour & & \\
Employee Green Behaviour & 0.924 & 0.922 \\
Task Performance & 0.844 & 0.837 \\
Core People Processes & 0.867 & 0.854 \\
Subscale WUOQ & & \\
Production and Efficiency & 0.816 & 0.803 \\
Work-Unit Climate & 0.904 & 0.903 \\
Future Growth & 0.851 & 0.844 \\
\hline
\end{tabular}

WUCQ, Work-Unit Competency Questionnaire; WUOQ, Work-Unit Outcome Questionnaire.

TABLE 4: The multidimensional McDonald's $\varpi$ and the stratified $\alpha$ for the WorkUnit Performance Questionnaire subscales for which the unidimensionality assumption had not been corroborated.

\begin{tabular}{lll}
\hline Subscale & McDonald's $\varpi$ & Stratified $\boldsymbol{\alpha}$ \\
\hline Subscale WUCQ & 0.790983472 & 0.865997172 \\
Effort & 0.760957393 & 0.826328631 \\
$\begin{array}{l}\text { Counterproductive work } \\
\text { behaviour }\end{array}$ & \\
Subscale WUOQ & 0.889982021 & 0.928322774 \\
Employee satisfaction & 0.800228225 & 0.876299697 \\
Market standing & 0.818683711 & 0.866399197 \\
\hline High performance culture &
\end{tabular}

WUCQ, Work-Unit Competency Questionnaire; WUOQ, Work-Unit Outcome Questionnaire. 
satisfactory stratified $\alpha$ estimates. The stratified $\alpha$ consistently returned substantially higher reliability estimates than the multidimensional McDonald's $\varpi$.

\section{Item parcels}

As previously discussed, all items produced satisfactory results in the item and dimensionality analysis. Therefore, all of the items were included in the item parcels. These parcels were made through calculating the mean of the items in the parcel. Two methods were used to identify the items that make up the relevant parcels. Firstly, random assignment (odd and even items) was used in instances when the unidimensionality assumption was corroborated. Thereafter, items were paired in accordance to their factor loadings in the pattern matrix in the instances when the unidimensionality assumption was not supported. The parcels acted as indicator variables of the latent variables in the process of fitting the proposed organisational work-unit measurement model and comprehensive LISREL models.

\section{Multivariate normality}

The premise that the indicator variables, in a continuous data set, have a multivariate normal distribution is fundamental in extracting estimates for freed model parameters through maximum likelihood. The statistics suggest that the indicator variables in both the WUCQ and WUOQ are not univariate normal $(p<0.05)$. When fitting measurement models to a continuous data set, the standard estimation technique used by LISREL 8.8 presumes multivariate normality. Thus, it was necessary to try and normalise the univariate distribution through PRELIS. However, the position that the data follows a multivariate normal distribution for the WUCQ, WUOQ and WUPQ was found to be invalid, so the null hypothesis had to be rejected. Therefore, robust maximum likelihood estimation was used to analyse the normalised data set in order to generate estimates for the freed parameters in the WUCQ, WUOQ and WUPQ measurement models.

\section{Fitting the Work-Unit Competency Questionnaire, Work-Unit Outcome Questionnaire and Work-Unit Performance Questionnaire measurement models}

Each item in the WUCQ and WUOQ scales was designed for the purpose of reflecting the standing of an organisational unit on a specific latent competency or outcome only. A detailed discussion of the various fit indices for the measurement model is provided in Seland (2019). Initially, the WUCQ measurement model returned an inadmissible solution with the loading of EGB_2 on the latent EGB competency exceeding unity and EGB_2 displaying a negative measurement error variance. The model successfully converged with an admissible solution when the loadings of EGB_1 and EGB_2 were fixed to 0.95. It is acknowledged that this is a methodological weakness that erodes the confidence in the WUCQ measurement model. Furthermore, the exactfit null hypothesis had to be rejected $\left(\chi^{2}=82.418 ; p<0.05\right)$ and the likelihood of observing the Root Mean Square Error of Approximation (RMSEA) sample estimation (0.0471) under the close-fit null hypothesis was adequately great enough (0.56367) to not reject the close-fit null hypothesis. Each one of the indicator variables were statistically significant $(p<0.05)$ in their loading on the relevant latent variables that they were intended to emulate. Lastly, the loading of CWB_2 on counterproductive work competency was the only factor loading that was less than the cut off at 0.71 in the completely standardised factor loading matrix.

For the WUOQ measurement model, the exact-fit null hypothesis was rejected $\left(\chi^{2}=52.036 ; p<0.05\right)$ and the chance of detecting the RMSEA sample estimate (0.0505) under the close-fit null hypothesis (0.459) was adequately great enough in order to not reject the close-fit null hypothesis. Furthermore, the indicator variables loaded on the latent variables they were intended to emulate $(p<0.05)$. The second production and efficiency item parcel and the second HPC parcel on the latent production and efficiency and HPC outcome variables, respectively, were the only factor loadings less than 0.71 in the completely standardised factor loading matrix.

The exact-fit null hypothesis was furthermore rejected $\left(\chi^{2}=\right.$ 336.833; $p<0.05)$ and the likelihood of detecting the RMSEA sample estimate (0.0510) under the close-fit null hypothesis (0.425) was satisfactorily great enough to not reject the closefit null hypothesis in the WUPQ measurement model. CWB_2 (0.50922) and HPC_2 (0.68529) were slightly below 0.71 in the completely standardised factor loadings. These aforementioned statistics are in agreement with the notion that the construct referenced inferences, taken from the dimension scores of the WUPQ, are construct valid. Moreover, these findings indicated that the operationalisation of the latent variables comprising the WUPQ structural model was generally fruitful.

\section{Discriminant Validity of the Work-Unit Performance Questionnaire measurement model}

The intention of the WUCQ, WUOQ and WUPQ subscales was to operationalise latent variables that are connected but qualitatively different. The investigation is to determine if the items designed to reflect each latent performance dimension was successful in differentiating the latent workunit dimensions. The latent variable inter-correlations were statistically significant $(p<0.05)$ in the WUPQ measurement model. Of the $78 \phi_{\mathrm{ij}}$ correlations, three exceeded the 0.90 critical value, and 17 were within 0.8 and 0.899 . Furthermore, for the 20 correlations greater than 0.8 , the $95 \%$ confidence interval for $\phi_{13,5}$ contained the value of one (Seland 2019). The inclusion of unity suggests that the indicators of task performance and production and efficiency may have measured the identical variable twice or measured the shared variance of the variables. This is, of concern, as it was suggested that task performance impacts the production and efficiency of the work unit. Thus, in the case of a statistically significant $(p<0.05)$ path coefficient finding it would be unclear if the finding was as a result of the hypothesised relationship, or as a result of the low discriminant validity. Fortunately, the other item parcels successfully operationalised the latent variables enough to differentiate the latent variables as qualitatively unique and separate constructs. However, 
the authors are aware that it is inadequate to infer that the WUPQ, in the measurement of the outstanding latent variables in the work-unit performance construct, exhibits discriminant validity.

\section{Evaluating the fit of the structural model}

The internal structure created for the organisational workunit performance construct is, to some extent, key in determining its connotative meaning. LISREL 8.8 was utilised to determine the fit of the work-unit performance comprehensive LISREL model. An inadmissible output was generated as a significant $(p<0.05)$ negative structural variance estimation for satisfaction was obtained. This necessitated fixing the paths to satisfaction from core people process and climate respectively to 0.35 . This resulted in the model converging after 31 iterations; however, there was a negative structural error variance estimate and a negative measurement error variance for satisfaction, as well as the EGB_2 composite indicator variable, respectively, that resulted in an inadmissible solution. Fortunately, there was no statistically significant $(p>0.05)$ deviation from zero for the estimates of the structural variance and the measurement variance. Therefore, it is the position of the research that the negative sign of these estimates should not be interpreted, similarly to any other statistically insignificant $(p>0.05)$ parameter estimates that are admissible. A path diagram of the revised fitted WUCQ measurement model is shown in Figure 2 and the full spectrum of fit statistics is presented in Table 5 .

The exact-fit null hypothesis was rejected, as the SatorraBentler chi-square produced a statistically significant figure $(547.80257 ; p<0.05)$. There was a fair fit of the model in the sample of the study as the RMSEA value of 0.071026 suggests. The likelihood of producing the aforementioned RMSEA figure in this sample if the close-fit null hypothesis was true in the parameter, was small enough (0.00006) to question the close-fit null hypothesis and, therefore, it was also rejected $(p<0.05)$. The model exhibited adequate fit in the sample but not close fit to the parameter. The array of fit statistics, displayed in Table 5, point towards the fact that the comprehensive model replicated the observed covariance matrix to an adequate level of accuracy that justified the interpretation of the structural model and the resultant $\boldsymbol{\Gamma}, \mathbf{B}$ and $\boldsymbol{\Psi}$ parameter estimates.

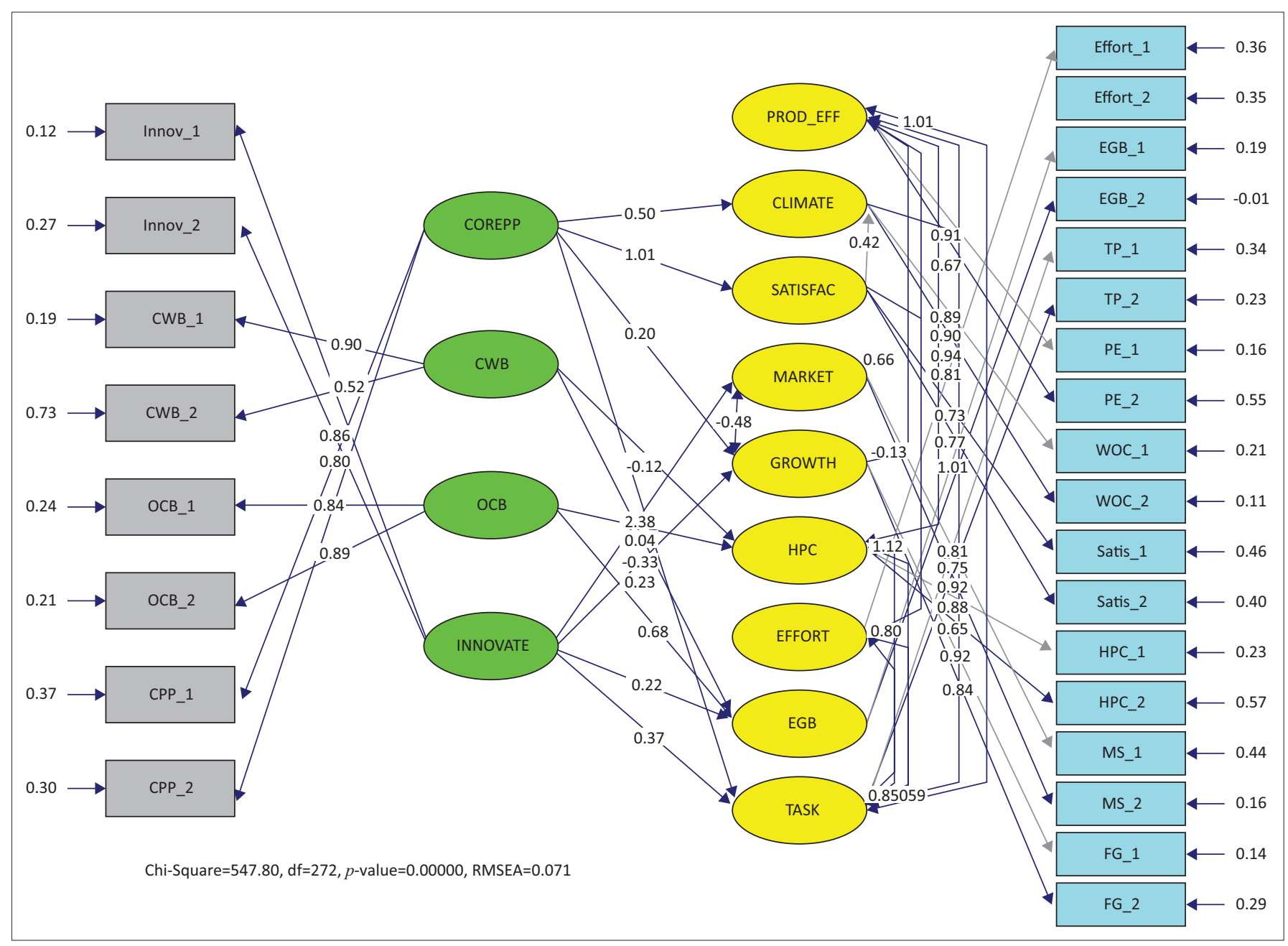

Source: Seland, J.M., 2019, 'The development and empirical testing of a revised Performance Index', Master's thesis, p. 251, Dept. of Industrial Psychology, Stellenbosch University.

$\mathrm{OCB}$, Organisational citizenship behaviour; EGB, employee green behaviour.CORE_PP, core people processes; CWB, counterproductive work behaviour; PROD_EFF, production and efficiency; SATIFAC, satisfaction; MARKET, market standing; GROWTH, future growth; HPC, high performance culture; TASK, task performance

FIGURE 2: Representation of the revised fitted comprehensive work unit performance LISREL model (completely standardised solution). 
TABLE 5: The goodness-of-fit statistics for the work-unit performance comprehensive LISREL model with paths from core people processes and climate to satisfaction were fixed to 0.35 .

Degrees of Freedom $\mathbf{=} \mathbf{2 7 2}$

Minimum Fit Function Chi-Square $=631.84471(p=0.0)$

Normal Theory Weighted Least Squares Chi-Square $=609.31360(p=0.0)$

Satorra-Bentler Scaled Chi-Square $=547.80257(p=0.0)$

Estimated Non-centrality Parameter (NCP) $=275.80257$

$90 \%$ Confidence Interval for $\mathrm{NCP}=(212.89789 ; 346.48500)$

Minimum Fit Function Value $=3.14351$

Population Discrepancy Function Value (F0) $=1.37215$

$90 \%$ Confidence Interval for $\mathrm{FO}=(1.05919 ; 1.72381)$

Root Mean Square Error of Approximation (RMSEA) $=0.071026$

$90 \%$ Confidence Interval for RMSEA $=(0.062403 ; 0.079609)$

$p$-value for Test of Close Fit (RMSEA $<0.05)=0.00006$

Expected Cross-Validation Index $(E C V I)=3.51146$

90\% Confidence Interval for ECVI $=(3.19850 ; 3.86311)$

ECVI for Saturated Model $=3.49254$

ECVI for Independence Model $=81.08765$

Chi-Square for Independence Model with 325 Degrees of Freedom = 16246.61752 Independence AIC = 16298.61752

Model AIC $=705.80257$

Saturated $\mathrm{AIC}=702.00000$

Independence CAIC = 16410.63248

Model CAIC $=1046.15572$

Saturated CAIC $=2214.20196$

Normed Fit Index (NFI) $=0.96628$

Non-Normed Fit Index (NNFI) $=0.97930$

Parsimony Normed Fit Index (PNFI) $=0.80870$

Comparative Fit Index $(\mathrm{CFI})=0.98268$

Incremental Fit Index (IFI) $=0.98273$

Relative Fit Index (RFI) $=0.95971$

Critical N (CN) $=121.78359$

Root Mean Square Residual (RMR) $=0.035620$

Standardised RMR $=0.057000$

Goodness-of-Fit Index (GFI) $=0.81096$

Adjusted Goodness-of-Fit Index (AGFI) $=0.75606$

Parsimony Goodness-of-Fit Index (PGFI) $=0.62844$

Source: Seland, J.M., 2019, 'The development and empirical testing of a revised Performance Index', Master's thesis, pp. 251-252, Dept. of Industrial Psychology, Stellenbosch University. AIC, Akaike information criterion; CAIC, consistent Akaike information criterion.

\section{Interpretation of structural model parameter estimates}

The findings suggest that $50 \%$ (five of the 10) of the beta pathspecific hypotheses were statistically significant $(p<0.05)$. These statistically significant paths postulated that increased production and efficiency, displayed by the work unit, will better the market standing of the aforementioned unit; furthermore, better production and efficiency in the work unit will increase its HPC; the work unit that depicts a stronger market standing will further its future growth; greater satisfaction in the work unit will result in greater effort shown by the members; and better task performance in the work unit shall result in greater production and efficiency of that unit.

Furthermore, $50 \%$ (six of the 12) of the gamma pathspecific hypotheses were statistically significant $(p<0.05)$. These significant paths were: increased, core people processes will further the task performance displayed by the work unit; greater core people processes result in greater satisfaction of the work unit; a greater amount of $C W B$ will lessen the $E G B$ of the work unit; higher citizenship behaviour will result in more $E G B$; the innovation of a work unit will promote its future growth; and innovation will increase the propensity of $E G B$.

In total, support was found for 11 of the 22 hypothesised relationships. This constitutes moderate support for the construct validity of the construct-referenced inferences derived from the WUPQ. The strongest overall relationship was the impact of increased core people processes on task performance (innovation, effort, climate and HPC are held constant). Thereafter, the influence that production and efficiency has on HPC was the second greatest (CWB and $O C B$ held constant). Other notably strong impacts were task performance on production and efficiency; production and efficiency on market standing (innovation kept constant); satisfaction on effort; and core people processes on satisfaction (Seland 2019).

\section{Discussion}

\section{Managerial implications}

It is essential to understand what comprises work-unit performance, and validly acknowledge the structural manner in which the latent constructs of work-unit performance combine in order for HR practitioners to successfully improve work-unit performance. To develop and empirically test a comprehensive work-unit competency model, a validated measure of work-unit performance is needed. Moreover, to obtain formative feedback on the need for and the nature of the intervention, the baseline level of competence achieved by organisational work units needs to be consistently observed. Thereafter, to evaluate whether the interventions have been successful, HR practitioners must subsequently monitor the level of performance by organisational work units. Once again, this necessitates a universally valid, reliable and unbiased tool to assess work-unit performance.

The WUPQ provides a generic tool for practitioners to assess the performance of work units by evaluating the proposed work-unit behavioural competencies and outcome variables in a variety of industries. Once assessed, practitioners can identify the latent performance dimensions on which a work unit performs relatively poorly (or relatively well). Thereafter, they can identify the upstream variables that need improvement and the impact thereof on relevant performance dimensions through the use of the organisational work-unit performance structural model. It is acknowledged that the fundamental idea is not new. What is new, though, is that the WUPQ, combined with the work-unit performance structural model, provides the basis of a formal diagnostic framework that may assist and guide HR practitioners and industrial psychologists in diagnosing and treating specific work-unit performance problems.

However, because of the current model's exclusion of competency potential and situational latent variables, the diagnostic value of the current work-unit performance structural model is still very limited and it should be used with caution. The current work-unit performance structural model 
could, however, be used with some greater confidence to guide and monitor work-unit performance and to identify lower levels of work-unit performance on specific dimensions. Future research should identify these competency potential and situational variables and graft them onto the existing work-unit performancestructural model in order to create a comprehensive work-unit competency model in the sense that was alluded to earlier, that could empower practitioners to diagnose workunit performance symptoms with increased precision.

\section{Future research}

It is suggested that in future research the two measurement models in this study be fitted with single items as indicators. This is the best method to assess the construct validity of the construct-referenced inferences derived from a multiindicator measuring instrument (Little et al. 2014), since it does not offer any problematic item the opportunity to hide in a composite indicator. However, the sample size in the current study was too small for individual items to be used (Bentler \& Chou, 1987) .

As previously stated, competency modelling, in the sense that it was defined in this study, gains its value from the fact that it attempts to capture the complex nomological network of latent variables constituting and determining a phenomenon like work-unit performance. Developing such a comprehensive competency model is too large a project to complete in a single study. Hence, the current study excluded the competency potential and situational latent variables and focussed solely on the explication of the internal structure of the work-unit performance construct. The work-unit competency potential and situational latent variables that determine the level of work-unit performance that is achieved, should be added in future research. It is proposed that the PI variables identified by Spangenberg and Theron (2004), classified as competency potential variables in the current study, be used as a starting point. These include adaptability and capacity. In addition, communication and self-development ought to be contemplated as further work-unit latent competencies in the work unit performance construct.

\section{Limitations to this study}

There are a few limitations that need to be considered. First of all, the study only managed to collect a relatively small sample of work units which draws into question the generalisability of the findings (Bentler \& Chou, 1987). Further, the same data set was used to determine the psychometric properties of the instrument and empirically assess the measurement and structural model. Moreover, the small sample forced the use of composite indicator variables in the fitting of the measurement models which created the possibility that items that are cause for concern may have hidden in the item parcels that were created. This is a limitation, as it is important to determine how well each individual item reflects the latent work- unit performance dimension it was designated to represent in the assessment of the construct validity.
Discriminant validity is most stringently assessed by comparing the average variance extracted (AVE) for a different latent variable with the squared correlation $\left(\phi_{\mathrm{jk}}\right)$ between latent variable pairs. AVE indicates the average proportion of variance that latent variable $j$ explains in the indicator, which were designated to reflect it (Diamantopoulos \& Siguaw 2000; Farrell 2010). Acceptable discriminant validity exists if AVEj is greater than 0.50 and AVEj and AVEk are greater than $\phi_{j \mathrm{k}}{ }^{2}$ (Farrell 2010). The current study did not examine discriminant validity from this perspective. It would be best examined when the study is repeated on a larger sample with the individual items as indicator variables.

Furthermore, it was a methodological debatable decision to advance with the analysis of the goodness-of-fit statistics after negative structural variance of estimates was obtained. However, the authors are of the opinion that statistically insignificant $(p>0.05)$ parameter estimates ought to be interpreted in a consistent manner. The assessment of the statistical significance of these parameter estimates involves assessing the null hypothesis, which fixes the value of the parameter to zero in the population. This null hypothesis should not be challenged if the likelihood of the sample estimate, under the hypothesised conditions in the parameter, is greater than the critical value of 0.05 . Therefore, the value and sign of the parameter estimates should not be interpreted if it is found to be statistically insignificant. This position is supported by Chen et al. (2001) when they proposed:

[A] nonsignificant error variance estimate is consistent with the idea of sampling variability leading to the negative estimates. Our results suggest that the next step should be to re-estimate the model constraining the error variances to zero or a small positive number. (p. 504)

The WUCQ and WUOQ were both self-report questionnaires, Babbie and Mouton (2002) suggest that in this type of measuring-instrument common method variance may occur, causing conclusions derived by a researcher to be inflated.

Furthermore, a demographically limited group of participants were invited to take part in this study as a result of the use of Facebook to recruit participants. However, it provided the opportunity to recruit individuals from a multitude of industries and trades. Therefore, the data had less of a chance to be influenced by organisational factors (such as retrenchments) and reduced the possibility of contaminated inferences by the researcher. Lastly, Facebook provided the opportunity to gather a sample in a relatively quick period (Boers 2014).

\section{Concluding remarks}

Organisations have a purpose to serve society with products and services that adequately meet the requirements of its members. However, the resources on earth are limited and organisations must use them in a non-wasteful manner through efficiency and effectiveness. This is largely dependent on the performance of an organisation's personnel. The focus 
has traditionally been on the performance of the individual employee on each level of the organisation with little attention given to the effectiveness and efficiency of work units within an organisation.

The performance of the work unit is not the sum of the performance of its individual employees. The performance of both the individual employee and the performance of the organisational work unit must be consistently enhanced through carefully developed interventions. It is too costly for organisations to leave work performance to chance. In order to achieve this, it is essential to (1) have a valid conceptual grasp on what constitutes individual and work-unit performance, (2) use measuring instruments of individual and collective workunit performance that is psychometrically sound and (3) validly understand the way that competency potential and situational latent variables combine to determine the performance of the individual and the work unit in the workplace.

This research furthered the quest for these conditions by contributing to the achievement of points 1 and 2 . The primary objective of this research study was to re-conceptualise the work-unit performance construct and to re-operationalise the construct taking the PI of Spangenberg and Theron (2004) as this basic point of departure. Overall, the study achieved to some degree the overarching objective as both measurement models (WUCP \& WUOQ) showed close fit. Furthermore, reasonable structural model fit was found in the sample and support was found for 11 of the original 22 path-specific substantive hypotheses and for an additional hypothesis.

The desire of the researchers is that this study will encourage additional studies aimed at the development and empirical testing of a comprehensive organisational work-unit competency model. This comprehensiveness is hoped to illicit the cunning logic and psychological mechanisms that impact work-unit performance in order for it to be purposefully altered (Ehrenreich 1991).

\section{Acknowledgements Competing interests}

The author(s) declare that they have no financial or personal relationship(s) that may have inappropriately influenced them in writing this article.

\section{Author's contributions}

All authors contributed equally to this work.

\section{Ethical consideration}

Ethical approval was granted by the humanities research ethics committee of Stellenbosch University.

\section{Funding information}

This research received no specific grant from any funding agency in the public, commercial or not-for-profit sectors.
This research received no specific grant from any funding agency in the public, commercial or not-for-profit sectors.

\section{Data availability}

The data that support the findings of this study are available from the corresponding author, J.S., upon reasonable request.

\section{Disclaimer}

The views and opinions expressed in this article are those of the authors and do not necessarily reflect the official policy or position of any affiliated agency of the authors.

\section{References}

Andersson, D., Rankin, A. \& Diptee, D., 2017, 'Approaches to team performance assessment: A comparison of self-assessment reports and behavioral observer scales', Cognition, Technology \& Work 19, 517-528. https://doi.org/10.1007/ s10111-017-0428-0

Babbie, E. \& Mouton, J., 2002, The practice of social research, Oxford University Press, Cape Town.

Bartram, D., Robertson, I.T. \& Callinan, M., 2002, 'Introduction: A framework for examining organisational effectiveness', in I.T. Robertson, M. Callinan \& D. Bartram (eds.), Organisational effectiveness: The role of psychology (pp. 01-10). Chichester, UK: Wiley.

Bartram, D., 2005, 'The great eight competencies: A criterion-centric approach to validation', Journal of Applied Psychology 90(6), 1185-1203. https://doi. org/10.1037/0021-9010.90.6.1185

Bartram, D., 2006, The SHL universal competency framework, SHL White Paper, SHL Group, Thames Ditton.

Bentler, P.M. \& Chou, C.P., 1987, 'Practical issues in structural modelling', Social Methods and Research 16(1), 78-117. https://doi.org/10.1177/0049124187016001004

Boers, M., 2014, 'Empirical evaluation of the Steyn-Boers structural model of psychological wellbeing at work', unpublished Master's thesis, Dept. of Industrial Psychology, Stellenbosch University.

Campbell, J.P., 1990, 'Modelling the performance prediction problem in industrial and organisational psychology', in M.D. Dunnette \& L.M. Hough (eds.), Handbook of industrial and organisational psychology (pp. 687-732). Palo Alto, Consulting Psychologists Press, Inc.

Campbell, J.P. \& Wiernik, B.M., 2015, 'The modelling and assessment of work performance. The Annual Review of Organisational Psychology and Organisational performance. The Annual Review of Organisational Psychology and Organisational
Behaviour', 2, 47-74. https://doi.org/10.1146/annurev-orgpsych-032414-111427

Chen, F., Bollen, K.F., Paxton, P., Curran, P. \& Kirby, J.B., 2001, 'Improper solutions in structural equation models; causes, consequences and strategies', Sociological Methods and Research 29(4), 468-508. https://doi.org/10.1177/00491241010 29004003

Cockerill, A.P., Schroder, H.M. \& Hunt, J.W., 1993, Validation study into the high performance managerial competencies, Working Paper, London Business School, performanc
London.

Cronbach, L.J., 1951, 'Coefficient alpha and the internal structure of tests', Psychometrika 16, 297-334. https://doi.org/10.1007/BF02310555

Diamantopoulos, A. \& Siguaw, J.A., 2000, Introducing LISREL, Sage, London.

Du Toit, M., Du Toit, S. \& Hawkins, D., 2001, Interactive LISREL: User's guide, Scientific Software International, Lincolnwood IL.

Duckworth, A., Peterson, C., Matthews, M. \& Kelly, D., 2007, 'Grit: Perseverance and passion for long-term goals', Journal of Psychology and Social Psychology 92(6), 1087-1101. https://doi.org/10.1037/0022-3514.92.6.1087

Dunn, T.J., Baguley, T. \& Brunsden, V., 2014, 'From alpha to omega: A practical solution to the pervasive problem of internal consistency estimation', British Journal of Psychology 105(3), 399-412. https://doi.org/10.1111/bjop.12046

Ehrenreich, B., 1991, 'May 20, Science, lies and the ultimate truth', Essay, Time $137(20), 66$

Elkington, J., 1998, Cannibals with forks: The triple bottom line of 21st century business, New Society Publishers, Vancouver.

Farrell, A.M., 2010, 'Insufficient discriminant validity: A comment on Bove, Pervan, Beatty, and Shiu (2009)', Journal of Business Research 63, 324-327. https://doi. org/10.1016/j.jbusres.2009.05.003

Goss-Sampson, M.A., 2019, Statistical analysis in JASP: A guide for students, Centre for Science and Medicine in Sport \& Exercise: University of Greenwich, Greenwich.

Henning, R., 2002, 'An investigation into the internal structure of the unit performance construct as measured by the performance index (PI)', unpublished Master's thesis, Dept. of Industrial Psychology, Stellenbosch University.

Henning, R., Spangenberg, H. \& Theron, C., 2004, 'The internal structure of the unit performance construct as measured by the Performance Index (PI)', SA Journal of Industrial Psychology 30(2), 26-36. https://doi.org/10.4102/sajip.v30i2.153 
Huysamen, G., 2006, 'Coefficient alpha: Unnecessarily ambiguous; unduly ubiquitous', SA Journal of Industrial Psychology 32(4), 34-40. https://doi.org/10.4102/sajip. v32i4.242

Kaur, M. \& Kaur, K., 2017, 'Human resource management: The need of today' International Journal for Innovative Research in Multidisciplinary Field 3(6), 203-206.

Little, T.D., Rhemtulla, M., Gibson, K. \& Schoemann, A.M., 2013, 'Why the items versus parcels controversy needn't be one', Psychological Methods 18(3), 285-300. https://doi.org/10.1037/a0033266

Lopez-Cabrales, A. \& Valle-Cabrera, R, 2020, 'Sustainable HRM strategies and employment relationships as drivers of the triple bottom line', Human Resource Management Review 30(3), 100689. https://doi.org/10.1016/j.hrmr.2019.100689

Love, J., Selker, R., Marsman, M., Jamil, T., Dropmann, D., Verhagen, J. et al., 2019 'JASP: Graphical statistical software for common statistical designs', Journal of Statistical Software 88(2), 1-17. https://doi.org/10.18637/jss.v088.i02

McDonald, R.P., 1999, Test theory: A unified treatment, L. Erlbaum Associates, Mahwah, NJ.

Muthén, B. \& Kaplan, D., 1985, 'A comparison of some methodologies for the factor analysis of non-normal Likert variables', British Journal of Mathematical and Statistical Psychology 38, 171-189. https://doi.org/10.1111/j.2044-8317.1985. tb00832.x

Myburgh, D. \& Theron, C.C, 2014, 'The development and evaluation of a generic and non-managerial performance measure', Management Dynamics 23(1), 26-58.

Nicholson, N. \& Brenner, S.O., 1994, 'Dimensions of perceived organisational performance: Test of a model', Applied Psychology: An international review 43(1) 89-108. https://doi.org/10.1111/j.1464-0597.1994.tb00811.x

Ones, D.S. \& Dilchert, S., 2012, 'Employee green behaviors', in S.E. Jackson, D.S. Ones \& S. Dilchert (eds.), Managing HR for environmental sustainability, pp. 85-116, Jossey-Bass/Wiley, San Francisco, CA.

Ones, D.S., Wiernik, B.M., Dilchert, S. \& Klein, R., 2015, 'Pro-environmental behavior', in J.D. Wright (ed.), International Encyclopaedia of the social \& behavioral sciences, 2nd edn., vol. 19, pp. 82-88, Elsevier, Oxford.

Seland, J.M., 2019, 'The development and empirical testing of a revised Performance Index', Master's thesis, Dept. of Industrial Psychology, Stellenbosch University.
SHL, 2000, Inventory management competencies, viewed 22 October 2020, from www.shl.com/SHL/za/Products/Access_Competencies/360/360_List/IM.

SHL, 2011, The SHL universal competency framework, viewed 20 September 2016, from http://www.shl.com/assets/resources/White-Paper-SHL-UniversalCompetencyFramework.pdf.

Slabbert, B. \& Hoole, C., 2021, 'A competency framework for coaches working in coaching development centres', SA Journal of Industrial Psychology/SA Tydskrif vir Bedryfsielkunde 47(0), a1841. https://doi.org/10.4102/sajip.v47i0.1841

Slaper, T.P. \& Hall, T.J., 2011, 'The triple bottom line: What is it and how does it work', Indiana Business Review 86(1), 4-8.

Spangenberg, H.H. \& Theron, C., 2004, 'Development of a questionnaire for assessing work unit performance', SA Journal of Industrial Psychology 30(1), 19-28. https:// doi.org/10.4102/sajip.v30i1.134

SPSS (2018), IBM SPSS software, Retrieved December 15, 2018 from https://www. ibm.com/za-en/analytics/spss-statistics-software.

Statistics South Africa, (2016), Quarterly employment statistics. Pretoria: Statistics South Africa

Swart, M.M., 2013, 'The development and empirical evaluation of a comprehensive leadership - Unit performance structural model', unpublished Master's thesis, Dept. of Industrial Psychology, Stellenbosch University.

Theron, C.C., 1999, 'Psychometric implications of corrections for attenuation and restriction of range for selection validation research', Doctoral dissertation, Dept. of Industrial Psychology, Stellenbosch University.

Theron, C.C. \& Spangenberg, H.H., 2016, 'An assessment of the structural invariance of the Performance Index structural model', Management Dynamics 25(2), 17-20.

Umar, Z., Kenourgios, D. \& Papathanasiou, S., 2020, 'The static and dynamic connectedness of environmental, social, and governance investments: International evidence', Economic Modelling 93, 112-124. https://doi.org/10.1016/j.econmod.2020.08.007

Viswesweran, C. \& Ones, D.S, 2000, 'Perspectives on models of job performance' International Journal of Selection and Assessment 8(4), 216-226. https://doi. org/10.1111/1468-2389.00151

Widhiarso, W. \& Ravand, H., 2014, 'Estimating reliability coefficient for multidimensional measures: A pedagogical illustration', Review of Psychology 21(2), 111-121. 